\title{
Efeito da realidade virtual no equilíbrio corporal de idosos: Revisão Sistemática
}

\author{
Effect of virtual reality on balance of the elderly: Systematic review \\ Efecto de la realidad virtual en el equilibrio corporal de los ancianos: Una revisión sistemática
}

Recebido: 14/04/2021 | Revisado: 21/04/2021 | Aceito: 25/04/2021 | Publicado: 09/05/2021

\author{
Adélia Ramos Alves \\ ORCID: https://orcid.org/0000-0002-3640-6868 \\ ALF Pilates e Fisioterapia, Brasil \\ E-mail: adeliaramos@gmail.com \\ Kamila Garcez de Almeida \\ ORCID: https://orcid.org/0000-0001-9623-2540 \\ Faculdade Anhanguera do Rio Grande, Brasil \\ E-mail: kamilagarcez@gmail.com \\ Aryane Laiz da Silva \\ ORCID: https://orcid.org/0000-0002-2824-1442 \\ Universidade de Franca, Brasil \\ E-mail: aryane.laiz@hotmail.com \\ Isabela dos Santos Campos Prudente Pinheiro \\ ORCID: https://orcid.org/0000-0002-5726-2189 \\ Universidade do Grande Rio, Brasil \\ E-mail: isa_santos_campos@yahoo.com.br \\ Daniella Cristina dos Santos Silva \\ ORCID: https://orcid.org/0000-0003-2565-3481 \\ Faculdade Estácio de Sá de Goiás, Brasil \\ E-mail: fisio.daniellac@gmail.com \\ Luiz Felipe da Silva Figueiredo \\ ORCID: https://orcid.org/0000-0003-1434-742X \\ Conservatório Brasileiro de Música - Centro Brasileiro de Educação, Brasil \\ E-mail: lipe.figueiredo@globo.com
}

\begin{abstract}
Resumo
Processo de envelhecimento influencia as capacidades físicas dos idosos, tornando-os mais suscetíveis a quedas e limitações funcionais. Equilíbrio postural prejudicado aumenta o risco de quedas em idosos. Exercício com realidade virtual tem sido amplamente utilizado para o equilíbrio postural de treinamento. Objetivo: deste estudo é investigar o efeito dos exercícios físicos com realidade virtual no equilíbrio corporal de idosos. Metodologia: Para esta revisão sistemática foi utilizado o método PRISMA, selecionou artigos randomizados e controlados publicados até Janeiro de 2021, que utilizavam videogame como recurso para reabilitação de equilíbrio corporal em idosos. A base de dados utilizada foi PubMed. Resultados: Foram encontrados 316 estudos, após as fases de triagem selecionamos 7 estudos para esta revisão sistemática. Os estudos passaram por uma avaliação metodológica pela escala de JADAD. O tamanho de efeito intra-grupos $(\mathrm{ESw})$ variou de pequeno a muito grande $(0,23-2,41)$ para o grupo que utilizou a $\mathrm{RV}$ como intervenção, enquanto para o grupo controle o tamanho de efeito variou de trivial a grande $(0,00-1,98)$. No entanto o tamanho de efeito inter-grupos variou de trivial a perfeito $(0,00-5,87)$ mostrando um efeito maior para o uso da RV. Conclusão: Concluímos que o uso da RV para a reabilitação de equilíbrio corporal é benéfico para a população idosa.

Palavras-chave: Realidade virtual; Equilíbrio; Exergames; Idosos.
\end{abstract}

\begin{abstract}
The aging process influences the physical capacities of the elderly, making them more susceptible to falling and functional limitations. Impaired postural balance increases the risk of falls in the elderly. Virtual reality exercise has been widely used for postural balance training. Objective: this study is to investigate the effect of physical exercises with virtual reality on the body balance of the elderly. Methodology: For this systematic review, the PRISMA method was used, selecting randomized and controlled articles published until January 2021, which used video games as a resource for the rehabilitation of body balance in the elderly. The database used was PubMed. Results: 316 studies were found, after the screening phases we selected 7 studies for this systematic review. The studies underwent a methodological evaluation using the JADAD scale. The size of the intra-group effect (ESw) varied from small to very large (0.23-2.41) for the group that used VR as an intervention, while for the control group the effect size varied from trivial to large ( 0.00-1.98). However, the size of the inter-group effect varied from trivial to perfect (0.00-5.87) showing a greater effect for the use of VR. Conclusion: We conclude that the use of VR for rehabilitation of body balance is beneficial for the elderly population.
\end{abstract}

Keywords: Virtual Reality; Balance; Exergames; Elderly. 


\begin{abstract}
Resumen
El proceso de envejecimiento influye en las capacidades físicas de las personas mayores, haciéndolas más susceptibles a caídas y limitaciones funcionales. El cambio del equilibrio postural aumenta el riesgo de caídas de los ancianos. Los ejercicios con realidad virtual han sido ampliamente utilizados para el equilibrio postural de entrenamiento. El objetivo de este estudio es investigar el efecto de los ejercicios físicos con realidad virtual en el equilibrio corporal de los ancianos. Metodología: Para esta revisión sistemática fué utilizado el método PRISMA, seleccionando artículos aleatorios y controlados publicados hasta enero de 2021 que utilizaban los videojuegos como recurso para la rehabilitación del equilibrio corporal de los ancianos. La base de datos utilizada fué PubMed. Hemos encontrado 316 estudios, después de las fases de selección elegimos 7 estudios para esta revisión sistemática. Los estudios han pasado por una evaluación metodológica por la escala JADAD. El tamaño del efecto intragrupo (ESw) ha variado de pequeño a muy grande $(0,23-2,41)$ para el grupo que utilizó la RV como intervención mientras que para el grupo de control el tamaño del efecto ha variado de trivial a perfecto $(0,00-1,98)$. Sin embargo, el tamaño del efecto intergrupal ha variado de trivial a perfecto $(0,00-5,87)$ mostrando un efecto más grande para el uso de la RV. Conclusión: Hemos concluido que el uso de la RV para la rehabilitación del equilibrio corporal es beneficioso para la población anciana.
\end{abstract}

Palabras clave: Realidad virtual; Equilibrio postural; Exergames; Ancianos.

\title{
1. Introdução
}

No processo de envelhecimento ocorrem alterações no sistema sensório-motor (Sousa et al., 2011) que afetam o controle postural (Mazini Filho et al., 2010), o equilíbrio corporal e a força muscular, consequentemente alterando a marcha e aumentando o declínio funcional, além do risco de quedas (Benedetti et al., 2008; Charchat-Fichman et al., 2005; Chodzko-Zajko et al., 2009; Mazini Filho et al., 2010; Panel on Prevention of Falls in Older Persons, 2011) no idoso.

O equilíbrio corporal pode ser definido como habilidade de manter ou restaurar um estado de equilíbrio de um corpo em repouso ou atividade (Pollock et al., 2000; van Diest et al., 2013).Envolve a integração das informações sensoriais (visual, vestibular e somatossensorial) (Fleury et al., 2013); e está relacionado ao risco de quedas em idosos (Mazini Filho et al., 2010). A fraqueza muscular e o déficit no controle postural são importantes fatores preditores de quedas nos idosos (Panel on Prevention of Falls in Older Persons, 2011; Pollock et al., 2000; van Diest et al., 2013), estando associados às condições de saúde e habilidades funcionais do indivíduo (Clark et al., 2010).

A capacidade funcional é um fator chave a ser alcançado nas intervenções com o idoso, principalmente por estar relacionada ao risco de quedas (Panel on Prevention of Falls in Older Persons, 2011). Desse modo, intervenções compostas por exercícios específicos de equilíbrio, força muscular e marcha, podem ser eficazes para a melhora do controle postural e, consequentemente capacidade funcional (Chodzko-Zajko et al., 2009; Panel on Prevention of Falls in Older Persons, 2011). Entretanto, tratamentos convencionais, compostos por repetições de exercícios específicos para equilíbrio podem ser monótonos para o paciente (Bateni, 2012; Gil-Gómez et al., 2011), o que pode impactar no engajamento do indivíduo à intervenção. Nesse contexto, uma alternativa recentemente estudada é o uso dos exercícios com realidade virtual (RV) nos tratamentos e treinamentos para o equilíbrio corporal, que vem se tornando cada vez mais popular, devido aos resultados positivos, observados principalmente em pacientes com comprometimento neurológico (Monteiro Junior \& da Silva, 2012); e o baixo custo (Bateni, 2012). Portanto, o uso da RV associada aos exercícios para o equilíbrio corporal vem ganhando espaço no meio científico e clínico, como uma intervenção alternativa (Bateni, 2012; Duque et al., 2013; Franco et al., 2012; Jorgensen et al., 2013; Padala et al., 2012; Rendon et al., 2012).

Alguns estudos com realidade virtual e exercícios têm mostrado resultados de melhora do equilíbrio corporal de idosos (Bateni, 2012; Cordeiro et al., 2020; Duque et al., 2013; Franco et al., 2012; Jorgensen et al., 2013; Padala et al., 2012; Rendon et al., 2012). Entretanto, são muitas as limitações de grande parte dos estudos, como o tamanho da amostra, análises de pouca robustez estatística e resultados incoerentes (Goble et al., 2014). As diferentes metodologias utilizadas nos estudos dificultam a compreensão dos reais efeitos do uso dessa técnica para o equilíbrio de idosos. Além disso, apesar do efeito positivo dos exergames (interface de realidade virtual proporcionada por vídeo game e associada aos movimentos corporais) em indivíduos com déficit neuromotor (Monteiro Junior \& da Silva, 2012), até o momento são poucos os estudos robustos realizados com 
idosos saudáveis. Com isso, há necessidade de uma revisão qualitativa e quantitativa sobre o assunto para entender qual o efeito que a realidade virtual pode surtir no equilíbrio de idosos. Portanto, o objetivo deste estudo é investigar o efeito dos exercícios físicos com realidade virtual no equilíbrio corporal de idosos.

\section{Metodologia}

\section{Método}

A característica desse estudo é descritiva, através de uma Revisão Sistemática de natureza quanti-qualitativa. O método adotado para este revisão sistemática, foi o protocolo do PRISMA (Liberati et al., 2009) para obtenção da resposta sobre o efeito da realidade virtual no equilíbrio corporal de idosos.

\section{Elegibilidade dos Estudos}

Apenas foram considerados elegíveis para o presente estudo os artigos originais e experimentos controlados e randomizados (ECRs) que investigaram o efeito crônico do treinamento ou reabilitação com o uso de realidade virtual, neste caso sendo utilizado apenas através de videogames, com pelo menos um desfecho para o equilíbrio corporal de idosos ( $\geq 60$ anos), publicados até Janeiro de 2021, sem restrições de idioma.

\section{Estratégia de Busca}

Para a busca dos artigos foram utilizadas as seguintes bases de dados: PubMed e SciELO. Além dessas bases, foram utilizados artigos identificados nas listas de referências de outras revisões e/ou de estudos originais relacionados ao tema.

Na busca dos artigos foram utilizados os termos em inglês (Wii OR virtual reality OR exergames AND elderly OR older OR community-dwelling AND balance OR postural balance OR equilibrium) e português (Wii, realidade virtual, exergames, idosos e equilíbrio, equilíbrio postural). Os sinônimos dos referidos termos foram identificados utilizando o MeSHTerms (para as palavras em inglês) e o DeCS (para as palavras em português). Tal procedimento foi adotado para aumentar a possibilidade de resultados nas buscas dos artigos. Os termos foram identificados no título ou nos resumos dos artigos. Recursos adicionais foram utilizados de acordo com a disponibilidade da base de dados (Ex. PubMed: boleanos (AND, OR, title/abstract); filtros (tipo de estudo, estudos em humanos, clinical trials, randomized clinical trial)). Esse procedimento teve como objetivo não somente filtrar os resultados, mas cruzar os termos principais para a obtenção do máximo de estudos possíveis.

\section{Seleção dos Estudos}

Esta etapa respeitou duas fases: 1) leitura dos títulos e leitura dos resumos; 2) leitura do texto na íntegra. Na primeira fase foram selecionados estudos de acordo com elegibilidade dos estudos. Na leitura do texto na íntegra, foram observados os principais resultados, levando em consideração os dados descritivos (em média e desvio-padrão) e inferenciais (nível de significância ou intervalo de confiança). Os artigos duplicados nas bases de dados ou lista de referências foram excluídos.

\section{Extração de Dados}

Foram extraídos de cada estudo os resultados numéricos, para posterior análise. Para tal, foram utilizados a média e o desvio padrão dos momentos pré e pós-intervenção, referente aos grupos experimental e controle de cada estudo. Em seguida foi realizado o cálculo do tamanho do efeito (TE) intra (TEintra) e inter grupos (TEinter) (Hopkings, 2000). Os valores negativos obtidos foram elevados ao quadrado e, posteriormente obteve-se a raiz quadrada desses valores. Tal procedimento foi aplicado para corrigir os valores negativos do TE, transformando-os em positivos. Para qualificação dos resultados, foi utilizada a classificação proposta por Hopkins (Hopkings, 2000). Os estudos que não apresentaram médias e desvios padrão como dados principais, foram abordados apenas de forma qualitativa. 
Para classificação da qualidade dos estudos selecionados e redução de viés, foi utilizada a Escala Jadad (Jadad et al., 1996), que avalia os ensaios de acordo com três itens, com um ponto em cada questão: 1) randomizado; é dado um ponto adicional nesta questão, se o método de randomização for descrito corretamente; 2) duplo-cego; adicionado um ponto a mais se o método de mascaramento for descrito corretamente; e 3)descrição das perdas e exclusões de sujeitos, totalizando cinco pontos na escala. O estudo é classificado de má qualidade ao obter dois pontos ou menos na escala

\section{Resultados}

Foram encontrados um total de 316 estudos, conforme demonstra a Figura 1. Após as exclusões dos 13 estudos duplicados, foram selecionados 89 títulos, que após a primeira triagem, de acordo com os critérios de seleção dos estudos, restaram 37 possíveis trabalhos para a revisão. No término da segunda triagem 7 estudos foram incluídos nesta revisão sistemática (Cho et al., 2014; Franco et al., 2012; Jorgensen et al., 2013; Jung et al., 2015; Karahan et al., 2015; Park et al., 2015; Toulotte et al., 2012).

Os estudos selecionados foram avaliados metodologicamente através da escala de JADAD (Jadad et al., 1996), como demonstrado pela Tabela 1. Apenas 2 estudos (Jorgensen et al., 2013; Karahan et al., 2015) obtiveram bom resultado pela JADAD, com pontuação total de 3, no entanto apenas um estudo(Park et al., 2015) não conseguiu pontuação pela escala.

Figura 1. Fluxograma dos estudos selecionados.

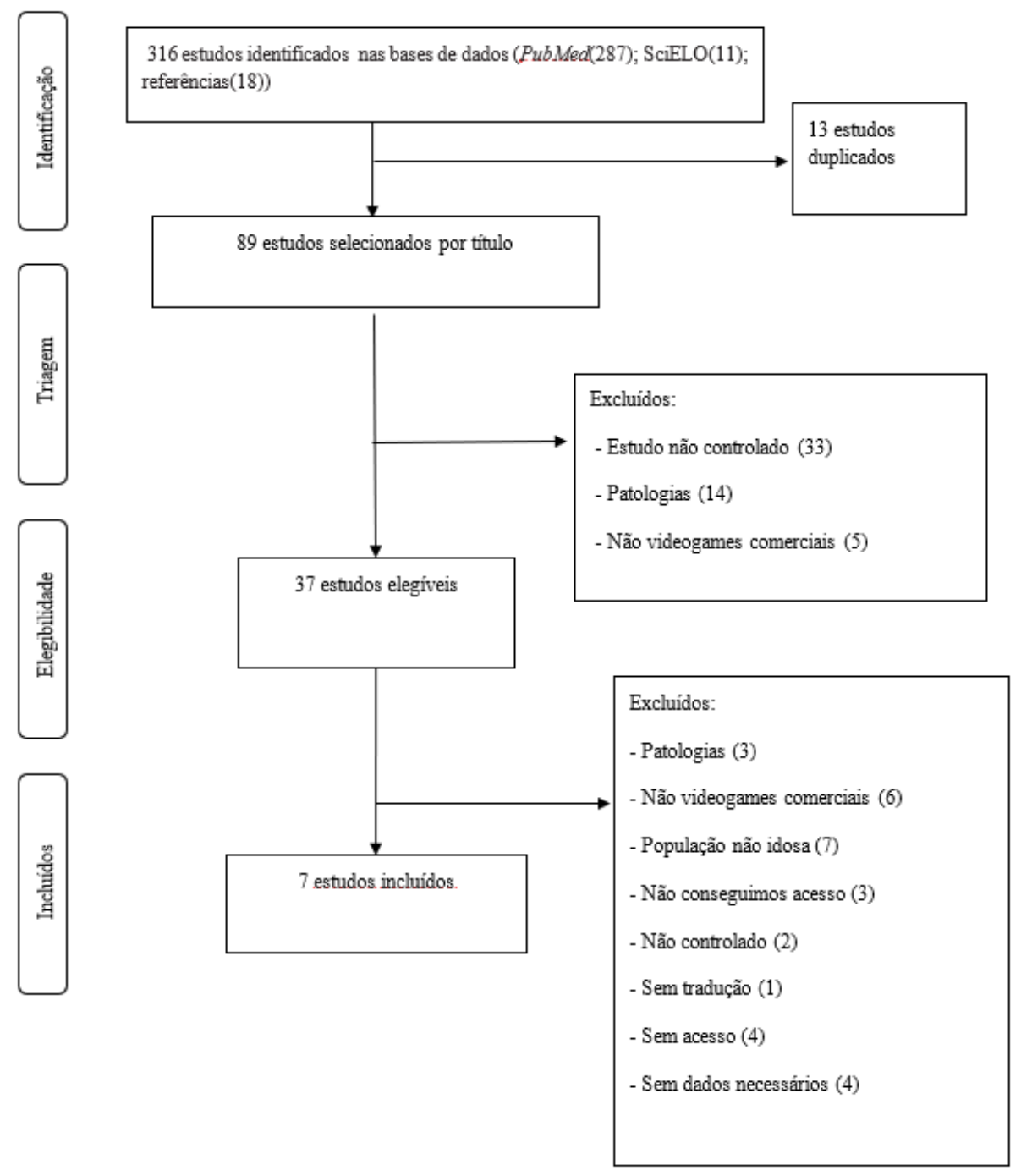

Fonte: Autores. 
Tabela 1. Qualidade Metodológica Dos Estudos Pela Escala Jadad

\begin{tabular}{|c|c|c|c|c|c|c|}
\hline \multicolumn{7}{|c|}{ Questões } \\
\hline Estudo & 1.a & $1 . b$ & 2.a & 2.b & 3 & Total \\
\hline Franco et al, 2012 & 1 & 0 & 0 & 0 & 1 & 2 \\
\hline Jorgensen et al, 2013 & 1 & 1 & 0 & 0 & 1 & 3 \\
\hline Toulotte et al, 2012 & 1 & 0 & 0 & 0 & 0 & 1 \\
\hline Cho et al, 2014 & 1 & 0 & 0 & 0 & 0 & 1 \\
\hline Park et al, 2015 & 0 & 0 & 0 & 0 & 0 & 0 \\
\hline Karahan et al, 2015 & 1 & 1 & 0 & 0 & 1 & 3 \\
\hline Jung et al, 2015 & 1 & 0 & 0 & 0 & 0 & 1 \\
\hline
\end{tabular}

Legenda: 1.a - Estudo foi descrito como randomizado?;; 1.b - randomização foi descrita e é adequada? 2.a - Descreveu método de mascaramento?; $2 . b$ Metodo de mascaramento foi adequado?; 3 Foram descritas as perdas e exclusões? Fonte: Autores.

As características de cada intervenção utilizadas pelos estudos nesta revisão são demonstradas pela Quadro 1. Seis estudos (Cho et al., 2014; Franco et al., 2012; Jorgensen et al., 2013; Jung et al., 2015; Park et al., 2015; Toulotte et al., 2012) utilizaram o videogame da Nintendo Wiiß e apenas um estudo (Karahan et al., 2015) utilizou o Xbox 360 Kinect@, todos estes tipos de intervenção necessitam que o idoso se movimente para que possa interagir com os jogos, podendo simular uma movimentação de algum esporte específico ou movimentos específicos para atingir os objetivos de cada jogo. Para os grupos controles as intervenções variam entre os estudos, três estudos (Jung et al., 2015; Karahan et al., 2015; Park et al., 2015) utilizaram um controle positivo colocando os idosos para realizarem exercícios variados, enquanto 2 estudos (Cho et al., 2014; Franco et al., 2012) não utilizaram intervenção alguma e 2 deles (Jorgensen et al., 2013; Toulotte et al., 2012) utilizaram atividades como assistir televisão ou usar uma palmilha. O tempo de intervenção varia entre os estudos também, de 10 minutos à 1 hora de atividade.

Os dados quantitativos e o tamanho de efeito de cada estudo são demonstrados na Tabela 2. O tamanho de efeito intergrupos, varia entre os estudos de trivial à perfeito. Para os cálculos de tamanho de efeito intra-grupos o grupo Wii não demonstrou nenhum resultado trivial, diferente do grupo controle. 
Research, Society and Development, v. 10, n. 5, e36510515057, 2021

(CC BY 4.0) | ISSN 2525-3409 | DOI: http://dx.doi.org/10.33448/rsd-v10i5.15057

\begin{tabular}{|c|c|c|c|c|c|c|}
\hline \multicolumn{7}{|c|}{ Quadro 1. Protocolos De Treino De Cada Estudo Selecionado. } \\
\hline Autor/Ano & Idade $($ Mean \pm SD) & $\mathbf{N}$ & Jogos & $\begin{array}{c}\text { Característica de } \\
\text { intervenção e } \\
\text { controle } \\
\end{array}$ & $\begin{array}{l}\text { Testes de } \\
\text { equilíbrio }\end{array}$ & Resultados \\
\hline \multirow[t]{2}{*}{ Franco et al, 2012} & Wii Fit $(79,8 \pm 4,7)$ & 11 & $\begin{array}{l}\text { Jogos do Wii 10-15 minutos (Soccer } \\
\text { Heading, Ski jumping, Ski slalom, wii } \\
\text { tightrope, table tilt, balance bubble) + } \\
\text { exercícios de força em casa. }\end{array}$ & \multirow[t]{2}{*}{$\begin{array}{l}\text { 2x por semana } \\
\text { durante } 3 \text { semanas }\end{array}$} & \multirow[t]{2}{*}{$\begin{array}{l}\text { BERG } \\
\text { Tinetti }\end{array}$} & \multirow[t]{2}{*}{$\begin{array}{c}\text { Todos os grupos melhoraram os } \\
\text { testes de equilíbrio. }\end{array}$} \\
\hline & Controle $(76,9 \pm 6,3)$ & 10 & Sem intervenção & & & \\
\hline \multirow[t]{2}{*}{ Jorgensen et al, 2013} & Wii Fit $(75,9 \pm 5,7)$ & 27 & $\begin{array}{l}\text { Jogos do Wii } 35 \pm 5 \text { minutos (Table Tilt, } \\
\text { Slalom Ski, Perfect 10, Tightrope Tension, } \\
\text { Penguin Slide, Standing Rowing Squat) }\end{array}$ & \multirow[t]{2}{*}{$\begin{array}{l}\text { 2x por semana } \\
\text { durante } 10 \\
\text { semanas }\end{array}$} & \multirow{2}{*}{$\begin{array}{c}\text { TUG } \\
\text { FES-I } \\
\text { Média da } \\
\text { velocidade de } \\
\text { deslocamento } \\
\text { do centro de } \\
\text { pressão }\end{array}$} & \multirow[t]{2}{*}{$\begin{array}{l}\text { Incrementaram TUG e FES-I no } \\
\text { grupo Wii, mas sem diferença na } \\
\text { velocidade do centro de pressão. }\end{array}$} \\
\hline & Controle $(73,7 \pm 6,1)$ & 30 & $\begin{array}{l}\text { Palmilhas de EVA (controlados através de } \\
\text { telefonemas nas semanas } 3,6 \text { e } 9 \text { ) }\end{array}$ & & & \\
\hline \multirow[t]{2}{*}{ Toulotte et al, 2012} & Wii Fit $(72,2 \pm 8,6)$ & 9 & $\begin{array}{l}1 \text { hora de jogos do Nintendo Wii (Heading } \\
\text { Soccer, Ski jumping, yoga, downhill skiing, } \\
\text { game balls, tightrope Walker) }\end{array}$ & \multirow[t]{2}{*}{$\begin{array}{l}\text { 1x por semana } \\
\text { durante } 20 \\
\text { semanas. }\end{array}$} & \multirow[t]{2}{*}{$\begin{array}{l}\text { Teste unipodal } \\
\text { Tinetti } \\
\text { Wii Fit }\end{array}$} & \multirow[t]{2}{*}{$\begin{array}{c}\text { Diferença positiva para o grupo } \\
\text { do Wii na parte estática do } \\
\text { Tinetti e no Wii Fit test. }\end{array}$} \\
\hline & Controle $(71,8 \pm 8,0)$ & 9 & Assistir televisão e jogos de tabuleiro & & & \\
\hline \multirow{2}{*}{ Cho et al, 2014} & Wii Fit $(73,1 \pm 1,1)$ & 17 & $\begin{array}{l}\text { Jogos do Wii (Ski Slalom, Table Tilt, } \\
\text { Balance Bubble) } 10 \text { minutos cada jogo }\end{array}$ & \multirow{2}{*}{$\begin{array}{c}\text { 3x por semana } \\
\text { durante } 8 \text { semanas }\end{array}$} & \multirow{2}{*}{$\begin{array}{l}\text { Teste de } \\
\text { Romberg na } \\
\text { plataforma de } \\
\quad \text { força }\end{array}$} & \multirow{2}{*}{$\begin{array}{l}\text { Houve uma redução da área } \\
\text { percorrida durante o teste para o } \\
\text { grupo Wii. }\end{array}$} \\
\hline & Controle $(71,7 \pm 1,2)$ & 15 & Sem intervenção & & & \\
\hline
\end{tabular}

Fonte: Autores. 
Research, Society and Development, v. 10, n. 5, e36510515057, 2021

(CC BY 4.0) | ISSN 2525-3409 | DOI: http://dx.doi.org/10.33448/rsd-v10i5.15057

\begin{tabular}{|c|c|c|c|c|c|c|}
\hline \multicolumn{7}{|c|}{ Cont. Quadro 1. Protocolos De Treino De Cada Estudo Selecionado. } \\
\hline Autor/Ano & Idade $($ Mean \pm SD) & $\mathbf{N}$ & Jogos & $\begin{array}{l}\text { Característica de } \\
\text { intervenção e } \\
\text { controle }\end{array}$ & $\begin{array}{l}\text { Testes de } \\
\text { equilíbrio }\end{array}$ & Resultados \\
\hline \multirow[b]{2}{*}{ Park et al, 2015} & Wii $(66,5 \pm 8,1)$ & 12 & $\begin{array}{l}\text { Jogos do Wii (Soccer heading, Snowboard } \\
\text { slalom, table tilt) } 10 \text { minutes cada jogo }\end{array}$ & \multirow[b]{2}{*}{$\begin{array}{l}\text { 3x por semana } \\
\text { durante } 8 \text { semanas }\end{array}$} & \multirow{2}{*}{$\begin{array}{c}\text { Área } \\
\text { percorrida e } \\
\text { velocidade } \\
\text { média de } \\
\text { deslocamento } \\
\text { do centro de } \\
\text { pressão } \\
\text { TUG }\end{array}$} & \multirow[b]{2}{*}{$\begin{array}{l}\text { Todos os grupos demonstraram } \\
\text { melhora em todos os testes, } \\
\text { porém o grupo Wii mostrou uma } \\
\text { superioridade no TUG } \\
\text { comparado ao grupo controle }\end{array}$} \\
\hline & Controle $(65,2 \pm 7,9)$ & 12 & $\begin{array}{c}\text { Exercícios com bola: } 20 \text { minutos de } \\
\text { exercícios com movimentos pélvicos sentado } \\
\text { na bola suíça e } 10 \text { minutos de exercícios } \\
\text { deitado com os pés na bola, como levar as } \\
\text { pernas de um lado ao outro e depois realizar } \\
\text { elevação de quadril. }\end{array}$ & & & \\
\hline \multirow[b]{2}{*}{ Karahan et al, 2015} & Wii $(71,3 \pm+6,1)$ & 48 & $\begin{array}{c}30 \text { minutos de jogos do Xbox } 360 \text { Kinect } \\
\text { (Futebol americano, tênis, tênis de mesa, ski, } \\
\text { golfe, voleibol e boliche) }\end{array}$ & \multirow[b]{2}{*}{$\begin{array}{c}\text { 5x na semana } \\
\text { durante } 6 \text { semanas }\end{array}$} & \multirow[b]{2}{*}{$\begin{array}{l}\text { BERG } \\
\text { TUG }\end{array}$} & \multirow{2}{*}{$\begin{array}{c}\text { Todos os grupos demonstraram } \\
\text { melhora no BERG, porém no } \\
\text { TUG apenas o grupo Wii } \\
\text { demonstrou melhora após a } \\
\text { intervenção. No BERG o grupo } \\
\text { Wii após a intervenção } \\
\text { demonstrou superioridade } \\
\text { comparado ao grupo controle. }\end{array}$} \\
\hline & Controle $(71,5 \pm 4,7)$ & 42 & $\begin{array}{l}\text { Exercícios para serem realizados em casa } \\
\text { (alongamentos de isquitibiais, quadricipes, } \\
\text { cintura pélvica, e peitorais; exercícios de } \\
\text { força para grandes grupamentos e exercícios } \\
\text { de equilíbrios como semitandem, ficar nas } \\
\text { pontas dos pés, ficar com pés juntos e } \\
\text { unipodal) } 30 \text { minutos desses exercícios. }\end{array}$ & & & \\
\hline \multirow[b]{2}{*}{ Jung et al, 2015} & Wii $(74,3 \pm 2,1)$ & 8 & $\begin{array}{l}\text { Jogos do Wii (wakeboard, frisbee dog, jet } \\
\text { ski, canoe) }\end{array}$ & \multirow{2}{*}{$\begin{array}{l}\text { 2x por semana } \\
\text { durante } 8 \text { semanas }\end{array}$} & \multirow{2}{*}{$\begin{array}{l}\text { BERG } \\
\text { Alcance } \\
\text { funcional } \\
\text { TUG }\end{array}$} & \multirow{2}{*}{$\begin{array}{l}\text { O grupo wii demonstrou melhora } \\
\text { em todos os testes e superior } \\
\text { comparado ao grupo controle. }\end{array}$} \\
\hline & Controle $(73,6 \pm 2,4)$ & 8 & $\begin{array}{c}30 \text { minutos de exercícios de pontes (15 } \\
\text { segundos 3x cada posição) (elevação de } \\
\text { quadril, ponte frontal e lateral, com elevação } \\
\text { de pernas e braços). }\end{array}$ & & & \\
\hline
\end{tabular}

Fonte: Autores. 
Tabela 2. Dados Quantitativos E Tamanho De Efeitos Dos Estudos Selecionados.

\begin{tabular}{|c|c|c|c|c|c|c|c|c|c|c|c|c|c|}
\hline \multirow[b]{2}{*}{ Autor/Ano } & \multirow[b]{2}{*}{ Wii } & \multirow{2}{*}{ N } & \multirow[t]{2}{*}{ Variáveis } & \multicolumn{2}{|c|}{ Pré-intervenção } & \multicolumn{2}{|c|}{ Pós-intervenção } & \multicolumn{2}{|c|}{ ESw } & \multicolumn{2}{|c|}{ Classificação } & \multirow[b]{2}{*}{$\mathbf{E S b}$} & \multirow[b]{2}{*}{ Classif. } \\
\hline & & & & $\begin{array}{c}\text { Wii } \\
M( \pm D P)\end{array}$ & $\begin{array}{l}\text { Controle } \\
\text { M ( } \pm \text { DP) }\end{array}$ & $\begin{array}{c}\text { Wii } \\
M( \pm \text { DP })\end{array}$ & $\begin{array}{l}\text { Controle } \\
M( \pm D P)\end{array}$ & Wii & Controle & Wii & Controle & & \\
\hline \multirow{5}{*}{ Toulotte, 2012} & \multirow{5}{*}{9} & \multirow{5}{*}{9} & $\begin{array}{l}\text { Teste Unipodal (OA) } \\
\text { (números de toques } \\
\text { do pé suspenso no } \\
\text { chão) }\end{array}$ & $17,8(3,7)$ & $19(6)$ & $14,5(2,1)$ & $18,1(5)$ & 0,89 & 0,15 & M & $\mathrm{P}$ & 0,74 & $\mathrm{M}$ \\
\hline & & & $\begin{array}{l}\text { Teste Unipodal (OF) } \\
\text { (números de toques } \\
\text { do pé suspenso no } \\
\text { chão) }\end{array}$ & $19,8(5,1)$ & $26,1(9,3)$ & $15(5)$ & $30,4(7,7)$ & 0,94 & 0,46 & M & $\mathrm{P}$ & 2,14 & MG \\
\hline & & & Tinetti Estático (pts) & $15,2(0,83)$ & $16,1(0,43)$ & $13,2 *(0,67)$ & $16,9(0,99)$ & 2,41 & 1,86 & MG & $\mathrm{G}$ & 5,87 & $\mathrm{PF}$ \\
\hline & & & Tinetti Dinâmico (pts) & $42,(0,23)$ & $4,3(0,43)$ & $3,8(0,67)$ & $4,3(0,2)$ & 1,74 & 0,00 & $\mathrm{G}$ & $\mathrm{T}$ & 1,52 & G \\
\hline & & & Posição do CG (\%) & $-20,6(4,5)$ & $-18,9(5,7)$ & $-12,5^{*}(2,2)$ & $-17,6(2,1)$ & 1,80 & 0,23 & $\mathrm{G}$ & $\mathrm{P}$ & 1,00 & $\mathrm{M}$ \\
\hline \multirow{2}{*}{$\begin{array}{c}\text { Jorgensen, } \\
2013\end{array}$} & \multirow{2}{*}{27} & \multirow{2}{*}{30} & TUG (seg) & $10,3(3,8)$ & $11(5)$ & $9 *(3,2)$ & $10,9(5,1)$ & 0,34 & 0,02 & $\mathrm{P}$ & $\mathrm{T}$ & 0,43 & $\mathrm{P}$ \\
\hline & & & FES-I (pts) & $11,3(3,5)$ & $11,3(4,3)$ & $10,5^{*}(3)$ & $11,6(3,8)$ & 0,23 & 0,07 & $\mathrm{P}$ & $\mathrm{T}$ & 0,28 & $\mathrm{P}$ \\
\hline \multirow{2}{*}{ Franco, 2012} & \multirow{2}{*}{11} & \multirow{2}{*}{10} & BERG (pts) & $48,5(9,1)$ & $50,3(3,7)$ & $52 *(5,4)$ & $51,4 *(2,9)$ & 0,38 & 0,3 & $\mathrm{P}$ & $\mathrm{P}$ & 0,09 & $\mathrm{~T}$ \\
\hline & & & Tinetti (pts) & $25,8(4,3)$ & $25,8(1,8)$ & $26,7 *(2,4)$ & $26,8 *(1,8)$ & 0,21 & 0,56 & $\mathrm{P}$ & $\mathrm{P}$ & 0,03 & $\mathrm{~T}$ \\
\hline \multirow{2}{*}{ Cho, 2014} & \multirow{2}{*}{17} & \multirow{2}{*}{15} & Romberg OA (cm) & $95.1(10.7)$ & $99.6(41.6)$ & $44.9 *(6.6)$ & $114.5(51.2)$ & 1,14 & 0,09 & M & $\mathrm{T}$ & 0,68 & $\mathrm{M}$ \\
\hline & & & Romberg OF (cm) & $104.1(20.4)$ & $109.9(15.3)$ & $35.6 *(7.6)$ & $72.5(20.9)$ & 0,82 & 0,63 & M & M & 0,52 & $\mathrm{P}$ \\
\hline
\end{tabular}

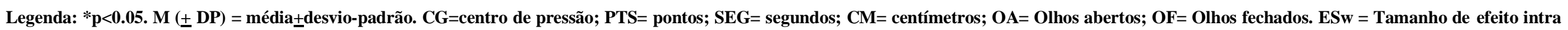
grupos, $\mathbf{E S b}=$ Tamanho de efeito inter grupos, $\mathrm{T}=$ trivial; $\mathrm{P}=\mathrm{Pequenol}, \mathrm{M}=$ Moderado, $\mathrm{G}=\mathrm{Grande}, \mathbf{M G}=\mathbf{M u i t o} \mathrm{Grande}, \mathrm{PF}=\mathrm{Perfeito}$.

Fonte: Autores. 
Research, Society and Development, v. 10, n. 5, e36510515057, 2021

(CC BY 4.0) | ISSN 2525-3409 | DOI: http://dx.doi.org/10.33448/rsd-v10i5.15057

Cont. Tabela 2. Dados Quantitativos E Tamanho De Efeitos Dos Estudos Selecionados.

\begin{tabular}{|c|c|c|c|c|c|c|c|c|c|c|c|c|c|}
\hline \multirow[b]{2}{*}{ Autor/Ano } & \multirow[b]{2}{*}{ Wii } & \multirow{2}{*}{$\begin{array}{l}\text { N } \\
\text { Controle }\end{array}$} & \multirow[t]{2}{*}{ Variáveis } & \multicolumn{2}{|c|}{ Pré-intervenção } & \multicolumn{2}{|c|}{ Pós-intervenção } & \multicolumn{2}{|c|}{ ESw } & \multicolumn{2}{|c|}{ Classificação } & \multirow[b]{2}{*}{ ESb } & \multirow[b]{2}{*}{ Classif } \\
\hline & & & & $\begin{array}{c}\text { Wii } \\
\text { M }( \pm \text { DP })\end{array}$ & $\begin{array}{l}\text { Controle } \\
\text { M }( \pm \text { DP })\end{array}$ & $\begin{array}{c}\text { Wii } \\
\text { M ( } \pm \text { DP })\end{array}$ & $\begin{array}{l}\text { Controle } \\
\text { M }( \pm \text { DP })\end{array}$ & Wii & Controle & Wii & Controle & & \\
\hline \multirow{3}{*}{ Park, 2015} & \multirow{3}{*}{12} & \multirow{3}{*}{12} & $\begin{array}{c}\text { Área percorrida do } \\
\text { CG }(\mathrm{mm})\end{array}$ & $54.8(5.9)$ & $55.2(4.1)$ & $41.6^{*}(4.1)$ & $47.1 *(9.2)$ & 2,24 & 1,98 & MG & G & 1,00 & M \\
\hline & & & $\begin{array}{l}\text { Velocidade média do } \\
\qquad \mathrm{CG}\left(\mathrm{mm}^{2}\right)\end{array}$ & $0.7(0.1)$ & $0.7(0.1)$ & $0.6^{*}(0.1)$ & $0.6^{*}(0.1)$ & 1,00 & 1,00 & $\mathrm{M}$ & M & 0,00 & $\mathrm{~T}$ \\
\hline & & & TUG $($ seg $)$ & $21.8(8.6)$ & $21.6(7.5)$ & $17.6 *(7.4)$ & $19.3 *(5.8)$ & 0,49 & 0,31 & $\mathrm{P}$ & $\mathrm{P}$ & 0,24 & $\mathrm{P}$ \\
\hline \multirow{2}{*}{ Karahan, 2015} & \multirow{2}{*}{48} & \multirow{2}{*}{42} & $\mathrm{BERG}$ (pts) & $49.8(3.8)$ & $49.4(3.7)$ & $54.9 *(2.6)$ & $51.1 *(4.1)$ & 1,34 & 1,70 & G & G & 0,91 & M \\
\hline & & & TUG (seg) & $8.7(1.7)$ & $8.6(1.7)$ & $8.0 *(16.4)$ & $8.6(1.8)$ & 0,41 & 0,00 & $\mathrm{P}$ & $\mathrm{T}$ & 0,41 & $\mathrm{P}$ \\
\hline \multirow{3}{*}{ Jung, 2015} & \multirow{3}{*}{8} & \multirow{3}{*}{8} & BERG (pts) & $39.1(1.4)$ & $39.8(1.8)$ & $41.0 *(2.2)$ & 38.4 (1.9) & 1,36 & 0,78 & G & M & 2,05 & MG \\
\hline & & & $\begin{array}{l}\text { Alcance Funcional } \\
\qquad(\mathrm{cm})\end{array}$ & $18.8(2.4)$ & $18.7(1.0)$ & $21.0 *(2.2)$ & $18.6(1.0)$ & 0,92 & 0,10 & M & $\mathrm{T}$ & 1,25 & G \\
\hline & & & TUG $($ seg $)$ & $12.1(2.3)$ & $12.0(1.2)$ & $9.4 *(2.3)$ & $12.0(1.6)$ & 1,17 & 0,00 & M & $\mathrm{T}$ & 1,47 & $\mathrm{G}$ \\
\hline
\end{tabular}

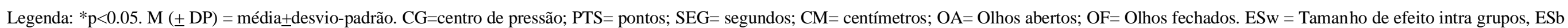
$=$ Tamanho de efeito inter grupos, $\mathrm{T}=$ trivial; $\mathrm{P}=$ Pequenol, $\mathrm{M}=$ Moderado, $\mathrm{G}=\mathrm{Grande}, \mathrm{MG}=$ Muito Grande, $\mathrm{PF}=$ Perfeito. Fonte: Autores . 


\section{Discussão}

Nossos resultados mostraram que o exercício usando RV promove um efeito positivo no equilíbrio postural em idosos. Muitos artigos originais têm mostrado que o VR aplicado a diferentes populações promove mudanças em muitas variáveis físicas como força muscular, a coordenação e o equilíbrio postural (Bateni, 2012; Costa et al., 2019; Duque et al., 2013; Franco et al., 2012; Gil-Gómez et al., 2011; Jorgensen et al., 2013; Monteiro Junior \& da Silva, 2012; Padala et al., 2017; Rendon et al., 2012; Sposito Letícia Aparecida Calderão, 2013). Os resultados nesta revisão mostraram uma melhoria no equilíbrio postural, usando a realidade virtual dos vídeos-games.

Esta revisão selecionou 7 estudos, que utilizavam à RV como forma de reabilitação para equilíbrio corporal de idosos. O uso da gameterapia, ou seja, o uso de videogame para reabilitação e treinamento da capacidade funcional de idosos, tem se mostrado uma alternativa para a prática da fisioterapia nesta população. Em um estudo (Monteiro-Junior et al., 2016) os autores demonstraram que o uso desta técnica em idosos institucionalizados incrementavam a capacidade funcional e cognitivas destes idosos, mostrando superioridade desta técnica comparado ao movimento tradicional, sem uso da RV. Corroborando também com os achados de (Stanmore et al., 2019), que demonstram o videogame como uma alternativa para melhorar o equilíbrio com potencial custo-benefício na população idosa, afirmaram também ótima alternativa para aumentar a aderência nas atividades físicas.

Os estudos diferem muito quanto ao uso desta técnica. Nesta revisão verificamos que o tempo de uso para a reabilitação variou de 10 minutos até 60 minutos; o volume de sessão também variou de 1 vez por semana até 5 vezes e a duração do programa durou de 3 semanas até 20 semanas. Esta grande variabilidade metodológica pode influenciar e justificar a falta de dados consistente na literatura ainda para esta técnica, dificultando para compilar os resultados de diferentes estudos para demonstrar o real efeito desta técnica. Nesta revisão realizamos o tamanho de feito para demonstrar um efeito clínico dos resultados, onde demonstramos grande variabilidade dos resultados, porém demonstramos 11 efeitos de moderado a perfeito, demonstrando o resultado benéfico desta técnica para a reabilitação de equilíbrio corporal de idosos.

$\mathrm{O}$ estudo que mostrou o pior resultado (ESb trivial e pequena $\mathrm{ESw}$ ) tem algumas características que devem ser consideradas (Franco et al., 2012). As pessoas que compuseram o grupo de Wii eram aparentemente mais velhos (74-91 anos) que o grupo controle (64-83 anos), mesmo que a idade média tenha sido semelhante $(79,8 \pm 4.7$ e $76.9 \pm 6.3)$. Os dados não foram analisados estatisticamente para determinar se os grupos eram homogêneos. Além disso, os autores citaram que dois participantes do grupo Wii necessitavam de auxilio para mobilidade, enquanto no grupo controle todos se locomoviam independentementes. É possível que o grupo controle possa ser mais ativo, assim gerando um aumento no volume de atividade física e influenciar os resultados.

Parece que a associação de RV a terapia convencional pode promover melhorias ainda mais significativas (Bateni, 2012; Toulotte et al., 2012). RV pode ser usado como uma alternativa por ter características lúdicas e motivacionais para melhorar as funções físicas e cognitivas, ao mesmo tempo(Monteiro-Junior et al., 2016, 2017; van Diest et al., 2013). O uso de feedback extrínseco pode ser uma ferramenta estimulante para ajustes posturais em tempo real que justifica a aplicação de exercícios com VR na reabilitação e exercício de treinamento para idosos(Monteiro Junior \& da Silva, 2012; Panel on Prevention of Falls in Older Persons, 2011).

Esta revisão mostrou que o uso dos videos-games como um dispositivo de realidade virtual pode promover efeito trivial à perfeito no equilíbrio postural. Entrada sensorial aumentada (feedback visual) com RV pode facilitar ajustes nos mecanismos de controle do corpo durante o exercício que cronicamente pode gerar uma melhor integração sensório-motor.

O presente estudo tem algumas limitações que devem ser consideradas. Havia poucos estudos elegíveis, e isto poderá prejudicar as conclusões sobre as evidências com relação a parâmetros de formação específica (duração, frequência, intensidade). Além disso, a qualidade metodológica dos artigos pode ter influenciado os resultados. 


\section{Conclusão}

Concluímos que a utilização da realidade virtual, através de videogames para a reabilitação do equilíbrio corporal de idosos é benéfica, promovendo melhora desta variável. No entanto esta pratica necessita ser mais explorada científicamente, pois a pequena quantidade de estudos que existem já vem mostrando resultados positivos para esta intervenção. Não podemos afirmar sua superioridade com outras praticas como exercícios já conhecidos, porém a RV se mostrou tão eficaz quanto aos exercícios praticados para este tipo de tratamento. Necessita de mais estudos, com metodologias adequadas e protocolos de intervenção mais homogêneos e melhor controle de volume de intervenção e jogos para que possamos obter conclusões mais definitivas.

\section{Referências}

Bateni, H. (2012). Changes in balance in older adults based on use of physical therapy vs the Wii Fit gaming system: a preliminary study. Physiotherapy, 98(3), 211-216. https://doi.org/10.1016/j.physio.2011.02.004

Benedetti, T. R. B., Borges, L. J., Petroski, E. L., \& Gonçalves, L. H. T. (2008). Atividade física e estado de saúde mental de idosos . In Revista de Saúde Pública. 42, 302-307.

Charchat-Fichman, H., Caramelli, P., Sameshima, K., \& Nitrini, R. (2005). Declínio da capacidade cognitiva durante o envelhecimento . In Revista Brasileira de Psiquiatria 27, 79-82

Cho, G. H., Hwangbo, G., \& Shin, H. S. (2014). The Effects of Virtual Reality-based Balance Training on Balance of the Elderly. Journal of Physical Therapy Science, 26(4), 615-617. https://doi.org/10.1589/jpts.26.615

Chodzko-Zajko, W. J., Proctor, D. N., Fiatarone Singh, M. A., Minson, C. T., Nigg, C. R., Salem, G. J., \& Skinner, J. S. (2009). Exercise and Physical Activity for Older Adults. Medicine \& Science in Sports \& Exercise, 41(7).

Clark, R. A., Bryant, A. L., Pua, Y., McCrory, P., Bennell, K., \& Hunt, M. (2010). Validity and reliability of the Nintendo Wii Balance Board for assessment of standing balance. Gait \& Posture, 31(3), 307-310. https://doi.org/10.1016/j.gaitpost.2009.11.012

Cordeiro, H. I. P., Rodrigues, A. C. de M. A., Alves, Rocha, M., Gatica-Rojas, V., Maillot, P., Pimentel, D. de M., Rezende, L. F. de, Rodrigues, V. D., Maia, M. de F. M., Carneiro, L. S. F., \& Monteiro-Junior, R. S. (2020). Exercise with active video game or strength/balance training? Case reports comparing postural balance of older women. Aging Clinical and Experimental Research, 32, 543-545. https://doi.org/https://doi.org/10.1007/s40520-019-01219-1

Costa, M., Vieira, L. P., Barbosa, E. O., Mendes Oliveira, L., Maillot, P., Ottero Vaghetti, C. A., Giovani Carta, M., Machado, S., Gatica-Rojas, V., \&, \& Monteiro-Junior, R. S. (2019). Virtual Reality-Based Exercise with Exergames as Medicine in Different Contexts: A Short Review. Clinical Practice and Epidemiology in Mental Health, 15.

Duque, G., Boersma, Loza-Diaz, Hassan, Suarez, H., Geisinger, Suriyaarachchi, Sharma, \& Demontiero. (2013). Effects of balance training using a virtualreality system in older fallers. Clinical Interventions in Aging, 257. https://doi.org/10.2147/CIA.S41453

Fleury, A., Mourcou, Q., Franco, C., Diot, B., Demongeot, J., \& Vuillerme, N. (2013). Evaluation of a Smartphone-based audio-biofeedback system for improving balance in older adults - A pilot study. 2013 35th Annual International Conference of the IEEE Engineering in Medicine and Biology Society (EMBC), 1198-1201. https://doi.org/10.1109/EMBC.2013.6609721

Franco, J. R., Jacobs, K., Inzerillo, C., \& Kluzik, J. (2012). The effect of the Nintendo Wii Fit and exercise in improving balance and quality of life in community dwelling elders. Technology and Health Care: Official Journal of the European Society for Engineering and Medicine, 20(2), 95-115. https://doi.org/10.3233/THC-2011-0661

Gil-Gómez, J.-A., Lloréns, R., Alcañiz, M., \& Colomer, C. (2011). Effectiveness of a Wii balance board-based system (eBaViR) for balance rehabilitation: a pilot randomized clinical trial in patients with acquired brain injury. Journal of NeuroEngineering and Rehabilitation, 8(1), 30. https://doi.org/10.1186/17430003-8-30

Goble, D. J., Cone, B. L., \& Fling, B. W. (2014). Using the Wii Fit as a tool for balance assessment and neurorehabilitation: the first half decade of "Wii-search." Journal of NeuroEngineering and Rehabilitation, 11(1), 12. https://doi.org/10.1186/1743-0003-11-12

Hopkings, W. G. (2000). A new view of statistics. Internet Society for Sport Science 2000.

Jadad, A. R., Moore, R. A., Carroll, D., Jenkinson, C., Reynolds, D. J., Gavaghan, D. J., \& McQuay, H. J. (1996). Assessing the quality of reports of randomized clinical trials: is blinding necessary? Controlled Clinical Trials, 17(1), 1-12.

Jorgensen, M. G., Laessoe, U., Hendriksen, C., Nielsen, O. B. F., \& Aagaard, P. (2013). Efficacy of Nintendo Wii training on mechan ical leg muscle function and postural balance in community-dwelling older adults: a randomized controlled trial. The Journals of Gerontology. Series A, Biological Sciences and Medical Sciences, 68(7), 845-852. https://doi.org/10.1093/gerona/gls222

Jung, D.-I., Ko, D.-S., \& Jeong, M.-A. (2015). Kinematic effect of Nintendo Wii(TM) sports program exercise on obstacle gait in elderly women with falling risk. Journal of Physical Therapy Science, 27(5), 1397-1400. https://doi.org/10.1589/jpts.27.1397

Karahan, A. Y., Tok, F., Taskin, H., Kucuksarac, S., Basaran, A., \& Yildirim, P. (2015). Effects of Exergames on Balance, Functional Mobility, and Quality of Life of Geriatrics Versus Home Exercise Programme: Randomized Controlled Study. Central European Journal of Public Health, 23 Suppl, S14-8. https://doi.org/10.21101/cejph.a4081 
Liberati, A., Altman, D. G., Tetzlaff, J., Mulrow, C., Gøtzsche, P. C., Ioannidis, J. P. A., Clarke, M., Devereaux, P. J., Kleijnen, J., \& Moher, D. (2009). The PRISMA statement for reporting systematic reviews and meta-analyses of studies that evaluate health care interventions: Explanation and elaboration. In $P L o S$ Medicine (Vol. 6, Issue 7, p. e1000100). John Wiley \& Sons. https://doi.org/10.1371/journal.pmed.1000100

Mazini Filho, M. L., Zanella, A. L., Aidar, F. J., Silva, A. M. S. da, Salgueiro, R. da S., \& Matos, D. G. de. (2010). Ativid ade física e envelhecimento humano: a busca pelo envelhecimento saudável. Revista Brasileira de Ciências Do Envelhecimento Humano., 7(1).

Monteiro-Junior, R. S., da Silva Figueiredo, L. F., Maciel-Pinheiro, P. de T., Abud, E. L. R., Braga, A. E. M. M., Barca, M. L., Engedal, K., Nascimento, O. J. M., Deslandes, A. C., \& Laks, J. (2016). Acute effects of exergames on cognitive function of institutionalized older persons: a single-blinded, randomized and controlled pilot study. Aging Clinical and Experimental Research. https://doi.org/10.1007/s40520-016-0595-5

Monteiro-Junior, R. S., Figueiredo, L. F. da S., Maciel-Pinheiro, P. de T., Abud, E. L. R., Engedal, K., Barca, M. L., Nascimento, O. J. M., Laks, J., \& Deslandes, A. C. (2017). Virtual Reality-Based Physical Exercise With Exergames (PhysEx) Improves Mental and Physical Health of Institutionalized Older Adults. Journal of the American Medical Directors Association, 18(5), 454.e1-454.e9. https://doi.org/10.1016/j.jamda.2017.01.001

Monteiro Junior, R. S., \& da Silva, E. B. (2012). Efetividade da reabilitação virtual no equilíbrio corporal e habilidades mo toras de indivíduos com déficit neuromotor: uma revisão sistemática. Revista Brasileira de Atividade Física e Saúde, 17(3).

Padala, K. P., Padala, P. R., Lensing, S. Y., Dennis, R. A., Bopp, M. M., Parkes, C. M., Garrison, M. K., Dubbert, P. M., Roberson, P. K., \& Sullivan, D. H. (2017). Efficacy of Wii-Fit on Static and Dynamic Balance in Community Dwelling Older Veterans: A Randomized Controlled Pilot Trial. Journal of Aging Research, 2017, 4653635. https://doi.org/10.1155/2017/4653635

Padala, K. P., Padala, P. R., Malloy, T. R., Geske, J. A., Dubbert, P. M., Dennis, R. A., Garner, K. K., Bopp, M. M., Burke, W. J., \& Sullivan, D. H. (2012). Wii-Fit for Improving Gait and Balance in an Assisted Living Facility: A Pilot Study. Journal of Aging Research, 2012, 1-6. https://doi.org/10.1155/2012/597573

Panel on Prevention of Falls in Older Persons, A. G. S. and B. G. S. (2011). Summary of the Updated American Geriatrics Socie ty/British Geriatrics Society Clinical Practice Guideline for Prevention of Falls in Older Persons. Journal of the American Geriatrics Society, 59(1), 148-157. https://doi.org/10.1111/j.15325415.2010.03234.x

Park, E.-C., Kim, S.-G., \& Lee, C.-W. (2015). The effects of virtual reality game exercise on balance and gait of the elderly. Journal of Physical Therapy Science, 27(4), 1157-1159. https://doi.org/10.1589/jpts.27.1157

Pollock, A. S., Durward, B. R., Rowe, P. J., \& Paul, J. P. (2000). What is balance? Clinical Rehabilitation, 14(4), 402-406. https://doi.org/10.1191/0269215500cr342oa

Rendon, A. A., Lohman, E. B., Thorpe, D., Johnson, E. G., Medina, E., \& Bradley, B. (2012). The effect of virtual reality gaming on dynamic balance in older adults. Age and Ageing, 41(4), 549-552. https://doi.org/10.1093/ageing/afs053

Sousa, R. F. de, Gazzola, J. M., Ganança, M. M., \& Paulino, C. A. (2011). Correlação entre equilíbrio corporal e capacidade funcional de idosos com disfunções vestibulares crônicas . In Brazilian Journal of Otorhinolaryngology (Vol. 77, pp. 791-798). scielo .

Sposito Letícia Aparecida Calderão, P. E. R. B. E. F. P. C. W. R. G. de S. F. F. da S. R. A. de. (2013). Experiência de trein amento com Nintendo Wii sobre a funcionalidade, equilíbrio e qualidade de vida de idosas. Motriz: Revista de Educação Física, 19, 532-540.

Stanmore, E. K., Mavroeidi, A., de Jong, L. D., Skelton, D. A., Sutton, C. J., Benedetto, V., Munford, L. A., Meekes, W., Bell, V., \& Todd, C. (2019). The effectiveness and cost-effectiveness of strength and balance Exergames to reduce falls risk for people aged 55 years and older in UK assisted living facilities: a multi-centre, cluster randomised controlled trial. BMC Medicine, 17(1), 49. https://doi.org/10.1186/s12916-019-1278-9

Toulotte, C., Toursel, C., \& Olivier, N. (2012). Wii Fit(R) training vs. Adapted Physical Activities: which one is the most appropriate to improve the balance of independent senior subjects? A randomized controlled study. Clinical Rehabilitation, 26(9), 827-835. https://doi.org/10.1177/0269215511434996

van Diest, M., Lamoth, C. J. C., Stegenga, J., Verkerke, G. J., \& Postema, K. (2013). Exergaming for balance training of elderly: state of the art and future developments. Journal of NeuroEngineering and Rehabilitation, 10(1), 101. https://doi.org/10.1186/1743-0003-10-101 